INSTITUTE FOR POLICY SCIENCE RESEARCH REPORT NO. 87-B-3

\title{
A COMPUTATIONAL METHOD FOR \\ SOLVING DEA PROBLEMS WITH \\ INFINITELY MANY DMUS \\ by
}

A. Charnes

K. Tone

March 24, 1987

Reprinted from Research Report CCS 561, Center for Cybernetic Studies, The University of Texas, at Austin. 
Research Report CCS 561

A COMPUTATIONAL METHOD FOR SOLVING DEA PROBLEMS WITH INFINITELY MANY DMUS

by

A. Charnes

K. Tone*

January 1987

- Graduate School for Policy Science, Saitama University, Urawa, Saitama 338, Japan

This research was partly supported by ONR Contracts N00014-86-C-0398 and N00014-82-K0295, and National Science Foundation Grant SES-8520806 with the Center for Cybernetic Studies, The University of Texas at Austin. Reproduction in whole or in part is permitted for any purpose of the United States Government.

\section{CENTER FOR CYBERNETIC STUDIES}

A. Charnes, Director

College of Business Administration, 5.202

The University of Texas at Austin

Austin, Texas 78712-1177

(512) $471-1821$ 
Research Report CCS 561

A COMPUTATIONAL METHOD FOR SOLVING

DEA PROBLEMS WITH INFINITELY MANY DMUS

by

A. Charnes and $\mathrm{K}$ Tone

\section{ABSTRACT}

Semi-inf inite programs as related to DEA with inf intely many DMUs will be solved by bisections within the framework of LPS. No gradient information Is needed, contrary to the usual NewtonRaphson type methods for solving seml-inf inite programs.

The rate of convergence is linear. The method has a stable convergency feature derived from the biscction rulc.

1. Problem

$2^{\circ}$ Outline of the Method

$3^{*}$ Details of the Method when $X$ is One-Dimensional

$4^{\circ}$ General Case 


\section{Problem .}

Solve the following LP with an inf inite set $X$ of DMUs for a suitable subset of elements typically designated by $x^{0}$ :

[Problem]

( $P$ )

$$
V_{p}=\max _{w} w^{\top} g\left(x^{0}\right) \quad: \quad x^{0} \in X
$$

subject to

$$
\begin{gathered}
w^{\top} g(x)=\mu^{\top}(-f(x)) \leq 0 \quad \text { for every } x \in X \\
\mu^{\top} f\left(x^{0}\right)=1 \\
w \quad z \in \cdot \dot{e} \\
\mu, \quad z \in \cdot e
\end{gathered}
$$

where $X$ : a compact convex set, $\operatorname{dim}(X)=L$,

$$
\begin{aligned}
& W \in R^{S}, \quad \dot{e}=(1,1, \ldots, 1)^{T} \in R^{S} \\
& \mu \in R^{m}, \quad e=(1,1, \ldots, 1)^{T} \in R^{m} \\
& g(x) \in R^{S} \quad: \text { continuous on } x \\
& f(x) \in R^{m} \quad: \text { continuous on } x \\
& \in: \text { a positive inf initesimal non-Archimedean quantity. }
\end{aligned}
$$

[Dual Problem]

(D) $\quad V_{D}=\min \left(Z-\epsilon \cdot e^{\top} S^{-}-\epsilon \cdot e^{T} S^{+}\right)$

subject to

$$
\begin{aligned}
& \sum_{x \in X} g(x) \lambda(x)-s^{-}=g\left(x^{0}\right) \\
& \sum(-f(x)) \lambda(x)-s^{+}=z\left(-f\left(x^{0}\right)\right)
\end{aligned}
$$

$\lambda(x) \geq 0$ : for every $x \in X$ and $\lambda(x)=0$ except for a finite number of points. $s^{-} \geq 0, s^{-} \in R^{s}$ $s^{+} 20, s^{+} \in R^{m}$. 
2 . Outline of the Method.

The method consists of three main parts: initial discretization, deletion and subdivision. The discretized problems are solved by the simplex method throughout the iterations.

Step 0. (Discretization)

The dual pair $(P)-(D)$ is discretized, i.e., the inf inite index set $X$ is replaced by a finite set. Let the finite set be $\left\{x^{1}, \ldots, x^{n}\right\}$. We call such sets grid.

Solve the resulting dual pair of linear programs $\left(P_{0}\right)-\left(D_{0}\right)$ by means of the simplex method.

$$
\left(P_{0}\right) \quad V_{p}=\max W^{\top} g\left(x^{0}\right)
$$

subject to

$$
\begin{aligned}
& W^{\top} g\left(x^{i}\right)+\mu^{\top}\left(-f\left(x^{i}\right)\right) \leq 0 \quad 1=1, \ldots, n \\
& \mu^{\top} f\left(x^{0}\right)=1 \\
& w r e \cdot \hat{\mathrm{e}} \\
& \mu z \text { e. e. } \\
& \left(D_{0}\right) \quad V_{D}=\min \left(Z \div C \cdot e^{T} S^{-}-\epsilon \cdot e^{T} S^{+}\right)
\end{aligned}
$$

subject to

$$
\begin{aligned}
& \sum_{i=1}^{n} g\left(x^{i}\right) \lambda_{i}-s^{-}=g\left(x^{0}\right) \\
& \sum_{i=1}^{n}\left(-f\left(x^{1}\right)\right) \lambda_{i}-s^{+}=z\left(-f\left(x^{0}\right)\right) . \\
& \lambda_{i} \geq 0 \\
& s^{-} \geq 0 \\
& s^{+}=0 .
\end{aligned}
$$


Let optimal solutions to $\left(P_{0}\right)$ and $\left(D_{0}\right)$ be

$$
w=\left(w_{1}, \ldots, w_{s}\right)^{\top}, \quad \mu=\left(\mu_{1}, \ldots, \mu_{m}\right)^{\top}
$$

and

$$
z, \lambda=\left(\lambda_{1}, \ldots, \lambda_{n}\right)^{\top}, \quad s^{-}=\left(s_{1}^{-}, \ldots, s_{s}^{-}\right)^{\top}, \quad s^{+}=\left(s_{1}{ }^{+}, \ldots, s_{m}{ }^{+}\right)^{\top} .
$$

Step 1. (Deletion)

Apply the 'Deletion rule' as explained later in Sections $3^{\circ}$ and $4^{\circ}$ to the grid $\left(x_{i}\right\}$.

Step 2. (Subdivision or Bisection)

Apply the 'Subdivision (bisection) rule' as explained in Sections $3^{\circ}$ and $4^{\circ}$ to the grid.

Step 3. (New $\left(P_{0}\right)$ and $\left.\left(D_{0}\right)\right)$

Formulate new dual LPS $\left(P_{0}\right)-\left(D_{0}\right)$ by deleting / augmenting constraints / variables to $\left(P_{0}\right)-\left(D_{0}\right)$. Solve them by the simplex method.

Step 4. (Convergence Check)

Stop the process if the subdivision parameter as explatned in Sections $3^{\circ}$ and $4^{\circ}$ becomes less than the tolerance. Otherwise go back to Step 1.

3.. Detalls of the Method When $X$ is One-Dimensional.

In this section, we will show detalls of the method in case $X$ is one dimensional. Cases with $\operatorname{dim}(x)>1$ will be discussed in Section $4^{\circ}$

3.1 Initial Discretization and Subdivision Parameter

Let the set $x$ be $[a, b] \subset R$ and arrange the grid $x_{0}, \ldots, x_{n}$ as

$$
a=x_{0}<x_{1}<\ldots<x_{n}=b
$$

where

$$
x_{i}=x_{0}+i(b-a) / n \quad(i=0, \ldots, n) .
$$

We def ine the subdivision parameter (or mesh size) $T$ to be

$$
T=(b-a) / n \quad \text { (the length of an interval) }
$$




\subsection{Solving $\left(D_{0}\right)$}

We solve the dual program $\left(D_{0}\right)$ by means of the simplex method. The reason for dealing with the dual program will be clarified later on. The optimal information related to the primal program is easily obtained from the optimal basis of $\left(D_{0}\right)$.

Let the optimal solution to $\left(P_{0}\right)$ and $\left(D_{0}\right)$ be

$$
w=\left(w_{1}, \ldots, w_{s}\right)^{\top}, \quad \mu=\left(\mu_{1}, \ldots, \mu_{m}\right)^{\top}
$$

and

$$
z, \lambda=\left(\lambda_{0}, \ldots, \lambda_{n}\right)^{\top}, s^{-}=\left(s_{1}^{-}, \ldots, s_{s}^{-}\right)^{\top}, s^{+}=\left(s_{1}{ }^{+}, \ldots, s_{m}{ }^{+}\right)^{\top} \text {. }
$$

\subsection{Deletion / Subdivision Rules}

Since the optimal solutions (3.4) - (3.5) solve the discretized problems, we have, at grid point $x_{i}$,

$$
w^{\top} g\left(x^{i}\right)+\mu^{\top}\left(-f\left(x^{i}\right)\right)=0 \quad \text { if } \lambda_{i}>0
$$

and

$$
w^{\top} g\left(x^{i}\right)+\mu^{\top}\left(-f\left(x^{1}\right)\right) \leq 0 \quad \text { if } \lambda_{i}=0
$$

However, it is not certain if the relations

$$
w^{\top} g(x)+\mu^{\top}(-f(x)) \geq 0
$$

hold for every $x \in X$.

Let $\quad \psi(x) \equiv w^{\top} g(x)+\mu^{\top}(-f(x))$.

The discrepancy $\delta(w, \mu)$ of $(w, \mu)$ is defined as

$$
\delta(w, \mu)=\max _{x \in[a, b]} \psi(x)
$$

An upper bound to $\delta(w, \mu)$ is given by

$$
\Delta=(F M|T| 2) / 8
$$

(Kortanek [1]), 
where $F$ is an upper bound to $w_{r}(r=1, \ldots, s)$ and $\lambda_{k}(k=1, \ldots, m)$

$$
M=\max _{x \in[a, b]}\left(\sum_{r=1}^{S}\left|g_{r}{ }^{\prime \prime}(x)\right|+\sum_{k=1}^{m}\left|f_{k}{ }^{\prime \prime}(x)\right|\right)<\infty
$$

and $T$ is defined by (3.3).

It is easy to see that if at two successive grid points $x_{i}$ and $x_{j}+1$, we have

$$
\psi\left(x_{i}\right)<-\Delta \text { and } \psi\left(x_{i+1}\right)<-\Delta,
$$

then it follows that

$$
\psi(x)<0 \quad \text { for every } x \in\left[x_{i}, x_{i+1}\right] .
$$

Thus, we have the deletion rule for grids.

[Deletion Rule]

If at three successive grid points $x_{1}, x_{1+1}$, and $x_{i+2}$, we have

$$
\psi\left(x_{i}\right)<-\Delta, \psi\left(x_{i+1}\right)<-\Delta \text { and } \psi\left(x_{i+2}\right)<-\Delta \text {, }
$$

then we delete $x_{i+1}$ and hence the whole interval $\left(x_{i}, x_{i+2}\right)$ from further consideratior.

Notice that the rule needs to be changed a little at the boundary points.

[Subdivision Rule]

We subdivide the remaining intervals by introducing a new grid at the mid-point of cach interval.

Thus, we have

$$
\begin{aligned}
& \text { new } T=T / 2 \quad \text { and } \\
& \text { new } \Delta=\Delta / 4 .
\end{aligned}
$$

[Remark 1] Usually it is not easy to determine $\Delta$ as defined by (3.11). In such a case, $\Delta$ (actually $-\Delta$ ) should be taken to be a threshold for deleting grid points. A smaller $\Delta$ (higher $-\Delta$ ) deletes more grid points. If $\psi(x)$ is well approximated by a quadratic curve at a local maximum, the relation (3.15) will generally hold af ter the subdivision. 
3.4 Solving the New LP

We delete the columns corresponding to the deleted grid points from the dual tableau and introduce new columns corresponding to the new grid points to the tableau. The new columns will be priced out by using the optimal dual basis of the preceding iteration and the primal simplex method will determine the new optimal solution.

\subsection{Convergence Check}

We stop the iterations if $T$ comes to satisfy, for some tolerance $T_{\text {tol }}$,

$$
T<T_{\text {tol }}
$$

[Remark 2] A typical process of subdivision (or bisection) is sketched in Fig. 1, where the curves represent $\psi(x)$ with $x$ as abscissa and the tolerance $(-\Delta)$ for each Iteration is given by the dashed line. 
Iteration I

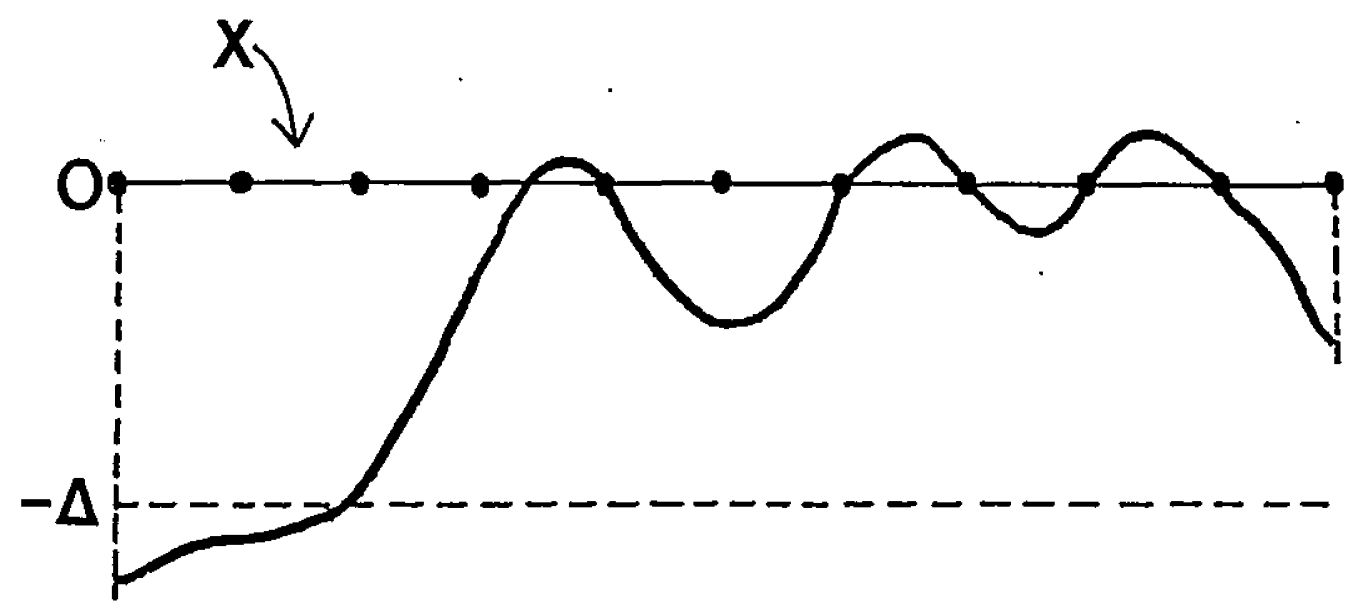

Itecation 2

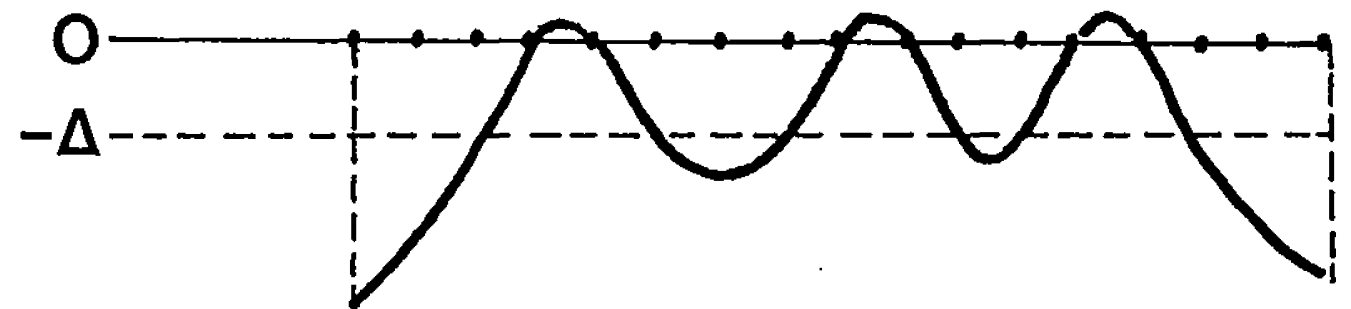

Iteration 3

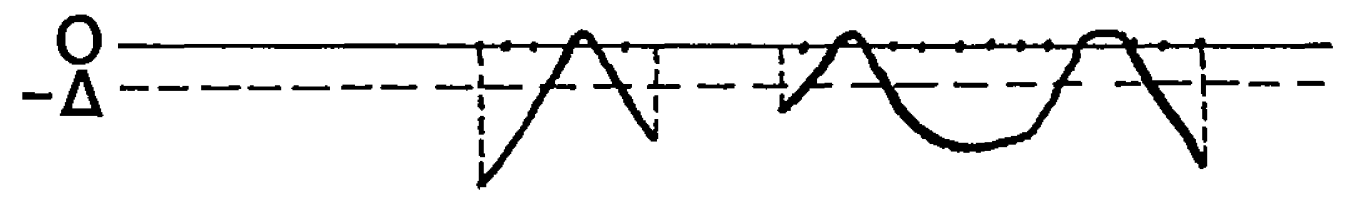

Iteration 4

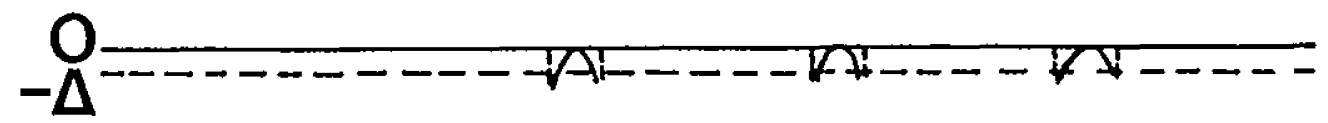

Fig. 1 
4. General Case.

In this section, we will deal with the dual pair of problems (P) - (D) when $X$ is a compact convex set with $L=\operatorname{dim}(x)>1$.

\subsection{Initial Discretization}

We discretize $X$ by using $L$ - dimensional cubes with edge length $T$. The mesh points are the initial grid points $\left(x^{1}, \ldots, x^{n}\right)$. The grid points are used to formulate $\left(P_{0}\right)$ and $\left(D_{0}\right)$, which are solved by the simplex method. Let the optimal solutions be $w, \mu, z, \lambda, s^{-}$, and $s^{+}$

\subsection{Deletion and Subdivision (BIsection) Rules}

Every grid has at most $2 \mathrm{~L}$ neighbors.

[Deleting Rule]

If the relation

$$
\psi(x) \equiv w^{\top} g(x)+\mu^{\top}(-f(x))<-\Delta
$$

holds at a grid and its neighbors, then we delete the center grid point and edges connecting the center with its neighbors from further consideration. $-\Delta$ is a threshold similar to (3.11) (see also [Remark 1]). For higher dimensional LS, it would be difficult to cstimate $\Delta$ by a formula such as (3.11). A practical way to estimate $\Delta$ is as follows: After the initial LPs are solved, we estimate the discrepancy $\delta(w, \mu)$ by sampling $x$ from $X$. The value will be used as the initial $\Delta$, which will be updated by dividing by 4 at each iteration.

[Subdivision Rule]

We divide the remaining edges by introducing a new grid at the mid-point of each edge. Thus we have

$$
\text { new } T=T / 2
$$

and

$$
\text { new } \Delta=\Delta / 4
$$


4.3 Solving New LPS and Checking Convergence

These steps are quite similar to those in the one-dimensional case as explained in subsections 3.4 and 3.5 .

[Remark 3] A typical subdivision process of the two-dimensional X is depicted in Fig. 2. 
Iteration 1

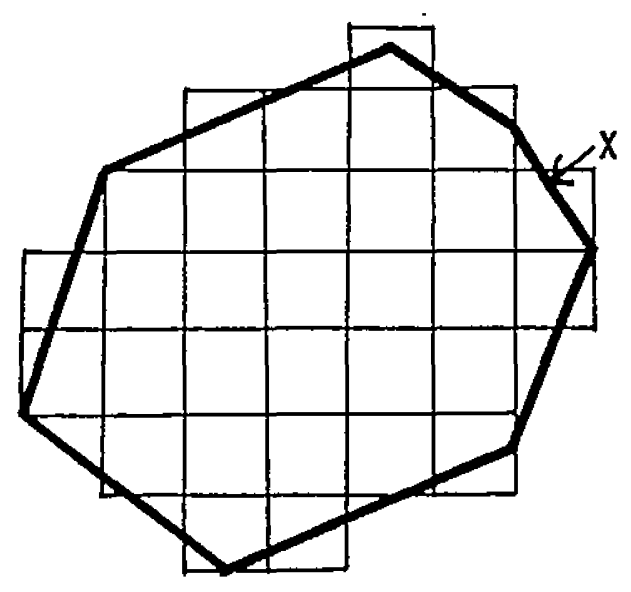

Iteration .2

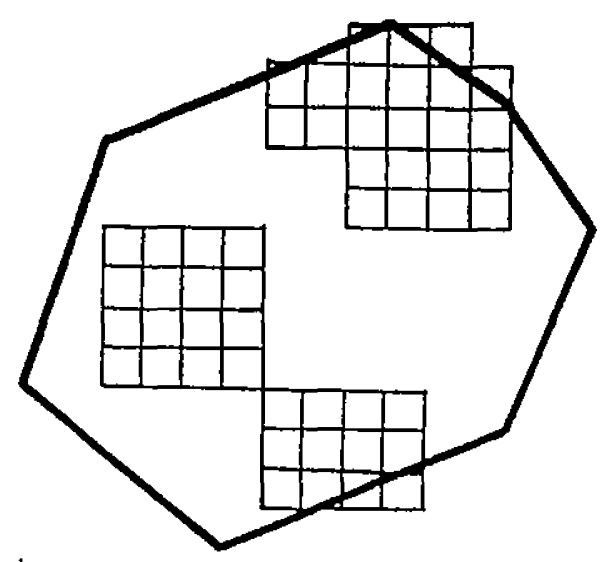

Iteration 3

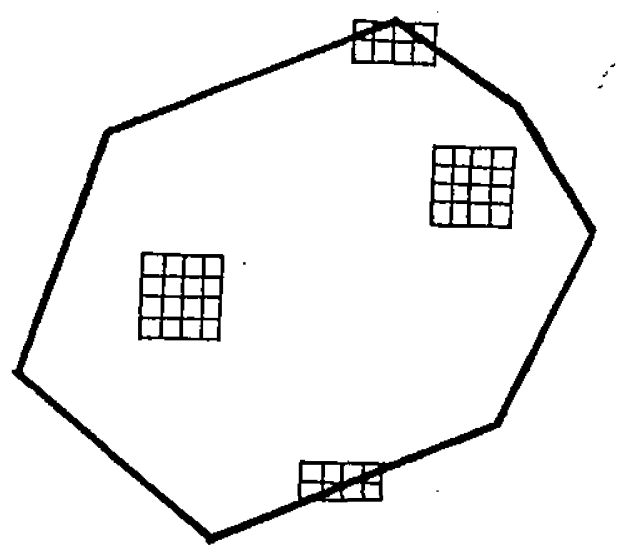

Fig. 2 


\section{REFERENCES}

[1] KORTANEK, K.O. "Interpolation and Error Bounds for Semi-Inf inite Programs and Solution of Nonlinear Systems of Equations" (1979) Manuscript. 